\title{
11 Incommensurability That Can(not) Be Ignored
}

\author{
Katie Steele
}

\section{Decide Based on That Which One Has an Opinion}

It seems natural and wise to make decisions on the basis of that which one has opinions, ignoring that which one has no clear opinion. For instance, consider the following very mundane decision problem:

Bike Route. I must decide which way to ride to work - whether the most direct route through the city center or a more convoluted route that is better connected by bike lanes and green spaces. There are a number of salient differences between these routes that matter for their evaluation, concerning such properties as their duration, safety, and aesthetics. There may well be other ways in which the two options differ, but I struggle to bring them to mind, let alone compare the bike routes with respect to them. For instance, how might my taking each route affect the well-being of all those I happen to interact with on the way and all those who interact with those people?

In framing the Bike Route decision problem and deliberating about what to do, it may seem reasonable that I focus just on those aspects or criteria for evaluating (pairs of) bike routes about which I have a clear opinion or preference relation with respect to the options. What is meant here by a "clear" opinion or preference relation is that I either (strictly) prefer one option to the other in the pair or else I am indifferent between the options in the pair. To lack a clear opinion or preference in this sense is then, let us say, to find the options incommensurable. (Here the term "incommensurable" is treated as synonymous with other terms of art in the literature, like incomparable or on a par. Any purported differences or ways in which these are distinct forms of lacking a regular preference relation between options are not important to the discussion here.) The thought is that the criteria under which the bike route options are incommensurable are precisely those that are less obvious or salient to me. While criteria under which options are incommensurable may sometimes 


\section{Katie Steele}

be salient, they are typically not, and that is perfectly reasonable, one might think, if such criteria are not important in decision-making.

My Bike Route deliberations seem entirely commonplace. If the aforementioned is an apt characterization of these deliberations, then it follows that we do commonly ignore in our decision-making that which we have no opinion about or criteria for which options are incommensurable since this is as mundane a decision problem as any. That is, it suggests that we invoke, in framing our decisions, some kind of "incommensurability independence" principle, whereby the relative value of options is independent of those aspects of the options that admit of no clear opinion or preference relation between them (namely, strict preference or indifference) such that they are incommensurable. Note that options may be incommensurable with respect to a criterion due to lack of opinion about the desirability of states of affairs by that criterion and/or lack of opinion about how likely it is that the options will result in these various states of affairs or realizations of the criterion. For instance, perhaps I cannot rank the bike routes with respect to the comfort of other riders because I cannot determine whether it is more or less or equally comfortable for a bike route to be more crowded. ${ }^{1}$ Otherwise (or in addition), perhaps I cannot rank the bike routes with respect to the comfort of other riders because I cannot assign precise probabilities to the relative size of the crowds on the bike routes on this particularly fresh spring day.

A principle akin to "incommensurability independence" is apparently defended by L. A. Paul in her (2014) book Transformative Experience. Paul claims that decisions involving transformative experiences, by definition, are decisions for which the options cannot be compared with respect to many aspects or criteria that one cares about (and thus also happen to be salient, in this case). Consider for instance the decision about whether to take on a new social role, such as whether or not to become a mother or whether or not to train to be a professional astronaut. The status quo and the role-change option may be difficult to compare with respect to criteria like "sense of personal achievement" and "level of anxiety." Nonetheless, Paul suggests that one can reasonably come to a choice based on the remaining aspects or criteria about which one does have an opinion, often simply the "surprise or adventure" that the options afford. ${ }^{2}$

In other discussions too, something like an "incommensurability independence" principle is floated as the only way to proceed in making rational decisions. For instance, Lenman (2000) raises a puzzle for those committed to (the relevance of) consequentialist ethical reasoning. The idea is that even the most foresighted agent has a sense of "cluelessness" about how the consequences of their options compare under the full myriad of ethically relevant aspects or criteria. One will easily become perplexed, for instance, in trying to predict which future persons may possibly exist and what these persons will go on to do and how good this 
would be, were some given option pursued. Greaves (2016: 312) says, in light of this problem, "In attempting to take consequences into account in practice in decision-making, we usually focus on those effects - let us call them 'foreseeable' effects - that we take ourselves to be able to foresee with a reasonable degree of confidence." One reading of this common wisdom (although not the one that Greaves favors) is that agents do and indeed rightly focus just on those aspects of the consequences of options that permit a clear comparison, in the sense used earlier, between them.

Finally, it seems that in the very framing of a decision problem, we routinely overlook aspects of options for which they are difficult to compare. Our reasoning concerns only the salient aspects of options or criteria for comparing them, and, as suggested earlier, the many aspects of options that are difficult to pin down and admit of no comparison between them are often not salient. So something like an "incommensurability independence" principle seems to play a role in shaping our very perception of a decision problem - the apparent starting point for our deliberations. Of course, if this really were the starting point for our deliberations it must by definition lie beyond rational principles. But one might suspect that what we typically regard as the starting point for our deliberations is actually the result of some prior (un)principled reasoning.

All told there is arguably a lot of implicit support for and allegiance to an "incommensurability independence" principle, whereby in comparing options, one simply ignores those aspects or criteria under which neither option is preferred to the other and nor is one indifferent between them. This chapter assesses whether this principle for comparing options is plausible. In fact, the chapter will assess just whether a weaker principle, entailed by the aforementioned, is plausible: a choice principle based on a kind of non-dominance relation that accommodates incommensurability between options on any given criterion. The non-dominance principle (or rather two versions of it, weak and strong) is introduced in Section 2. The principle is compared with a somewhat analogous principle in the literature on incommensurability and uncertainty owing to Caspar Hare (2010).

In Section 3, it is argued that neither of the two versions of the nondominance principle is plausible. The case against the strong version is relatively straightforward - it is easy to construct counterexamples. But the weak version too is subject to counterexamples, albeit of a special and more unusual form. Whether or not any of these counterexamples are genuine, however, might be open to challenge, especially in light of the debate inspired by Hare's analysis. I show in Section 4 that, despite the analogies, there are significant dis-analogies between the multi-criteria and uncertainty settings, and so the counterexamples retain their force. Finally, Section 5 returns to the question of whether we do routinely ignore aspects of our decisions for which we cannot compare options or whether our ordinary deliberations can be cast in a more reasonable light. 


\section{Katie Steele}

\section{Making the Principle(s) Precise}

To explore the form of reasoning suggested in the previous section, I will introduce a different decision problem, for which, by assumption, there is salient incommensurability between the options. That is, the options are incommensurable with respect to certain aspects of their consequences or criteria that are nonetheless salient (perhaps owing to the fact that one of the options is recognizably novel or unfamiliar). When incommensurabilities are explicitly tabled in this way, we can examine whether it is generally coherent to "ignore incommensurabilities" in one's reasoning.

The problem is a very simplistic climate change policy problem. The choice is between the status quo option of business-as-usual or inaction on climate change, and a geoengineering option. We will assume that for the (rather short-sighted) decision-maker at hand, there are only two aspects of the outcome, or two criteria, that matter for the comparison of the options: the welfare of the present generation (labeled "current") and the welfare of the subsequent generation (labeled "future"). These criteria are separable (in the sense that they amount to independent, additive contributions to the overall value of the options) and together exhaust all that matters for the decision-maker in comparing the options.

To add some color: the geoengineering option involves the current generation paying for this capability and the subsequent or future generation implementing it. The status quo is thus perceived to be better for the current generation than the geoengineering option since it does not involve costs associated with building geoengineering capability. Hence the utility associated with the former, $a$, is greater than the utility associated with the latter, $b$. The two options are, by assumption, incommensurable for the future generation - each has indeterminate utility for this criterion (compatible with incommensurability), represented by "ind." (In subsequent models of the problem, further assumptions will be introduced by way of making the indeterminate utilities more precise).

The informal choice principle of "ignoring incommensurabilities" recommends the status quo option in this decision problem. In comparing the options, we ignore the "future" criterion, as the options are incommensurable under this criterion - both business-as-usual and the deployment of geoengineering are, after all, very hard to evaluate, and ultimately compare, for the generation directly experiencing them. We then focus just on the "current" criterion, or what the options are like for the current generation. Under this criterion, the status quo option is preferred.

The reasoning just described in fact amounts to a special case of "ignoring incommensurabilities" - it involves no more than an appeal to (some kind of) dominance of one option over the other. That is, it appears we need only appeal to a choice principle that invokes the following rather compelling non-dominance relationship (the choice of terminology will be explained next): 
(Criterion-Wise) Competitive. An option is not worse than another option, and thus (criterion-wise) competitive, if, under every criterion, its outcome is not worse than that of the other option.

Only the status quo option is (criterion-wise) competitive. The status quo option is not worse than the geoengineering option for those criteria under which the options are incommensurable (here only "future") and it is moreover not worse than the geoengineering option (in fact it is better) under the remaining "current" criterion. As such, the status quo option is surely a permissible option, and plausibly uniquely so.

The choice principles just alluded to can in fact be formalized as weak and strong versions of what we might call "(criterion-wise) competitiveness," as follows:

Weak (Criterion-Wise) Competitiveness. If an option alpha is (criterion-wise) competitive with respect to all other alternative options, it is rationally permissible to perform alpha.

Strong (Criterion-Wise) Competitiveness. If an option alpha is (criterion-wise) competitive with respect to all other alternative options, it is rationally permissible to perform alpha, and it is not rationally permissible to perform an option beta that is not similarly (criterion-wise) competitive with respect to all other alternative options.

Both of these non-dominance or so-called competitiveness principles seem compelling, even the strong version. Moreover, they are analogues of non-dominance principles (across states) that have been recently entertained by Hare (2010) and defended by Bales et al. (2014). Indeed the terminology here follows that of Bales et al. (2014). ${ }^{3}$ One can restore the state-wise principles by replacing the aforementioned "criterion" with "state." Start with the very notion of a non-dominated or competitive option:

(State-Wise) Competitive. An option is not worse than another option, and thus (state-wise) competitive, if, under every state, its outcome is not worse than that of the other option.

This notion then features in the two choice principles (with labels roughly in keeping with those of Bales et al. (2014)):

Weak (State-Wise) Competitiveness. If an option alpha is (state-wise) competitive with respect to all other alternative options, it is rationally permissible to perform alpha.

Strong (State-Wise) Competitiveness. If an option alpha is (statewise) competitive with respect to all other alternative options, it is 


\section{Katie Steele}

rationally permissible to perform alpha, and it is not rationally permissible to perform an option beta that is not similarly (state-wise) competitive with respect to all other alternative options.

Despite the analogy with plausible state-wise, non-dominance choice principles, neither the strong nor the weak criterion-wise versions are themselves compelling. This will be demonstrated in the following section. We will return to why the two sets of choice principles - state-wise and criterion-wise, respectively - have differing status, due to important dis-analogies, later.

\section{Against the Criterion-Wise "Competitiveness" Principles}

We can interrogate the strong (criterion-wise) competitiveness principle by initially considering different versions or instantiations of the decision problem described in Table 11.1. This involves examining the different ways in which the options may be incommensurable under the second criterion ("future") and how this is weighed against the preference for the status quo under the first criterion ("present"). Assume that the (expected) utilities in Tables 11.2,11.3, and 11.4 are comparable and scaled in such a way that the two criteria are weighted equally. Indeterminate (expected) utility is modeled in terms of sets of (expected) utility functions; two options are incommensurable when the (expected) utility functions in the set do not agree on the ranking of the options. Finally, the overall (expected) utilities for the options are determined in the usual way, as a weighted average of the (expected) utilities for the options under the criteria.

Table 11.2 gives one instantiation of the more general geoengineering problem described in Table 11.1. The (expected) utilities $a$ and $b$ (where $a>b$ ) associated with the present outcomes are specified to be 0 and -100 , respectively. And the sets of (expected) utilities for the future generation

Table 11.1 A multi-criteria problem with explicit incommensurability

\begin{tabular}{lcl}
\hline & current & future \\
\hline status quo & $a$ & ind \\
geoengineering & $b$ & ind \\
\hline
\end{tabular}

Table 11.2 So far, so good for (criterion-wise) competitiveness

\begin{tabular}{lrl}
\hline & current & \multicolumn{1}{c}{ future } \\
\hline status quo & 0 & $\{-50,-50\}$ \\
geoengineering & -100 & $\{-500,40\}$ \\
\hline
\end{tabular}


outcomes represent incommensurability between these outcomes as required. While the status quo is expected to yield -50 utils for the future generation, the geoengineering option is expected to yield either -500 or 40. (For simplicity, let us assume this is a discrete set containing only two expected utility functions. ${ }^{4}$ ) However, once we take into account the current generation as well, the two options are not incommensurable. The status quo has expected utility of -25 (the result of $0.5 \times 0+0.5 \times-50$ ). Geoengineering has expected utility of $\{-300,-30\}$; that is, it either has expected utility of -300 (the result of $0.5 \times-100+0.5 \times-500$ ) or else -30 (the result of $0.5 \times-100+0.5 \times 40$ ). Both of these expected utilities for geoengineering are less than -25 and so the status quo option comes out as clearly preferable overall. This looks good even for the strong (criterion-wise) competitiveness principle. By this principle, the status quo option is uniquely permissible, in line with the numbers.

But we can easily see how the numbers might not have been so favorable for strong (criterion-wise) competitiveness. Consider the version of the geoengineering problem in Table 11.3, which is a slight adjustment of the previous version.

Again, the expected utility of the status quo is -25 . But this time, the expected utility of the geoengineering option is $\{-300,-20\}$. So by the standard method for determining the overall evaluation of options, the status quo and geoengineering options are incommensurable. According to one expected utility function, the status quo is better since $-25>-300$. But according to the other, the geoengineering option is better since -25 $<-20$. So it is not the case that the status quo is uniquely permissible, as per strong (criterion-wise) competitiveness.

Before moving on, it is important to address a worry about where the (expected) utility values in the above tables and those that follow come from. One might wonder what licenses any given representation of a decision problem. Surely the representation is derived from the agent's overall preference ordering, and what is at issue here are constraints on that very preference ordering. If strong (criterion-wise) competitiveness is a constraint on rational preferences, then Table 11.3 together with the stated calculus for determining the overall evaluation and thus ranking of the options simply does not represent a rational agent. It might be added that an objection to strong (criterion-wise) competitiveness must involve a demonstration that the principle conflicts with an alternative and even

Table 11.3 A prima facie violation of strong (criterion-wise) competitiveness

\begin{tabular}{lcl}
\hline & current & future \\
\hline status quo & 0 & $\{-50,-50\}$ \\
geoengineering & -100 & $\{-500,60\}$ \\
\hline
\end{tabular}




\section{Katie Steele}

more compelling principle of preference/choice. And that has not been provided here.

A preliminary response to this objection is simply to push the can a little down the road. For now, we are assuming that a rational agent entertaining a decision problem for which the criteria are separable and exhaustive of what matters to her and moreover admit of comparable (expected) utility measures, has overall preferences that can be represented as the weighted average of her expected utilities across the criteria. ${ }^{5}$ (We are further allowing that preferences may be incomplete under any given criterion, represented as sets of expected utilities, yielding sets of weighted average expected utilities overall.) The initial question is not whether strong or weak (criterion-wise) competitiveness should be regarded as a primitive or basic constraint on rational preferences. Rather, it is the more modest question of whether there is in fact a conflict between strong or weak (criterion-wise) competitiveness and the model of rational preference just described. And we see from Table 11.3 that there is indeed a conflict. This provides some reason to doubt strong (criterion-wise) competitiveness; whether we should rather rethink our supposed model of rational preference will be taken up in the next section.

One way to pose the lesson here is that when a pair of options are incommensurable for one or more criteria in a decision problem, on the model we are supposing, the incommensurability can "swamp" the overall evaluation of the options, even if one option is strictly preferred on the remaining criteria. It is only in special cases that the incommensurability of options on some criteria can be regarded as "minor," in the sense that the associated range of expected utilities does not undermine the strict preference for one option on the remaining criteria. Hence strong (criterion-wise) competitiveness is not generally consistent with the model of rational preferences in the multi-criteria setting that is supposed here.

Let us turn now to the weak (criterion-wise) competitiveness principle. Are there cases where, despite being (criterion-wise) competitive or nondominated, an option is nonetheless not permissible? The answer is "yes." Again, a small variation on the previous decision problem can show that even weak (criterion-wise) competitiveness is not generally consistent with the model of rational preference presupposed here.

In the new case, described in Table 11.4, the options are incommensurable under both criteria. Hence by weak (criterion-wise) competitiveness, both options are permissible. And yet, by the lights of our supposed model of rational preference, the geoengineering option here is not permissible. The geoengineering option will be either worse than the status quo for the current generation by 100 and yet better for the future generation by 40 , or it will be better than the status quo for the current generation by 20 , and yet worse for the future generation by 300 . As such, geoengineering is overall determinately worse. Indeed, the status quo has an overall expected utility 
of -25 while geoengineering has (determinately less) overall expected utility of $\{-55,-165\}$; that is, it either has an overall expected utility of -55 (the result of $0.5 \times-100+0.5 \times-10$ ), or else it has overall expected utility of -165 (the result of $0.5 \times 20+0.5 \times-350$ ).

Admittedly, this is a rather peculiar case, insofar as the agent's attitudes go. The peculiarity is the strange complementarity between the agent's preference attitudes across the two criteria on all candidate expected utility functions: Either geoengineering is worse for the current generation but better for the future generation, or else geoengineering is better for the current generation and yet worse for the future generation. This pattern is crucial for geoengineering coming out determinately worse overall. (All that is further required for that result is that geoengineering is not sufficiently better for the current/future generation to compensate for just how much worse it is for the other generation.) In particular, the other two logical possibilities - whereby geoengineering is worse on both criteria or else better on both criteria - must not be live candidates for the agent.

What kind of story might we tell to make the aforementioned model seem plausible? Let the geoengineering option involve the current generation doing a trial implementation and addressing any problems that arise before the future generation does a full implementation. Perhaps the agent is divided between two evaluations because they cannot assign precise probabilities to the possibility that crucial problems will be identified in the trial. If the problems are identified, then the geoengineering option will be worse for the current generation but slightly better, let's say, for the future generation; if the problems are not identified, then the geoengineering option will be better for the current generation but much worse for the future generation when hitherto unforeseen problems arise that are nonetheless expected to be highly detrimental. Assume the agent has a rather strange belief state: for whatever reason, she thinks either the crucial problems being identified is a certainty, or else the crucial problems not being identified is a certainty (and nothing in between). Hence there are two possible expected utility representations across the two criteria, as per Table 11.4. One can appreciate from this sketchy narrative that decision problems like those described in Table 11.4 are rather unusual. Nonetheless, they are a conceptual possibility. Indeed, a simpler problem in the same vein will be introduced in the next section.

Table 11.4 A prima facie violation of weak (criterion-wise) competitiveness

\begin{tabular}{lrc}
\hline & \multicolumn{1}{c}{ current } & future \\
\hline status quo & $\{0,0\}$ & $\{-50,-50\}$ \\
geoengineering & $\{-100,20\}$ & $\{-10,-350\}$ \\
\hline
\end{tabular}




\section{Katie Steele}

\section{Comparison with the State-Wise Analogues}

It was briefly noted earlier that there is room for dispute about the status of the state-wise competitiveness principles. Hence one might presume that there should equally be room for dispute about the status of their criterion-wise counterparts. As such, one might think that the supposed model of rational (multi-criteria) preference in Section 3 is more contentious than first meets the eye such that the conflict with the (criterion-wise) competitiveness principles is far from devastating for the latter. A closer look at the extent of the analogy between the state-wise and the criterionwise principles does not support this optimism about the latter, however. That is what we turn to now: a demonstration that the two versions of competitiveness are not on a par as constraints on rational preference.

We will focus on the weak form of the competitiveness principles since these are lesser constraints on rational preference. The last section presented a prima facie case that not even weak (criterion-wise) competitiveness is a plausible constraint on rational preference. The following decision problem - "Two Transparent Boxes" - presents this case in simpler terms, (even) more contrived as it may be. Indeed, the problem will play a useful role in our subsequent discussion because it has the same tabular form - with the exception of the labeling and meaning of the columns - as the decision problem introduced by Casper Hare (2010) entitled "Two Opaque Boxes." Hare's problem inspired much further discussion of constraints on rational preference where incommensurability and risk are concerned, including Bales et al.'s (2014) defense of what we here refer to as weak (state-wise) competitiveness.

Here is the analogue to Hare's decision problem (with crucial similarities to the final geoengineering case, as will be elaborated next):

Two Transparent Boxes. You show me items $A$ and $B$, two dollar coins, two twins Pablo and Penelope, and two transparent boxes, the contents of which must be distributed between the twins in specified ways. I consider $A$ and $B$ to be incommensurable with respect to both Pablo and Penelope's welfare. Perhaps $A$ is a ticket to the opera while $B$ is a ticket to an A-league sports game. (Moreover, I consider the twins to derive the same welfare from the same items.) The twins' welfare is all that matters to me and the welfare of each matters equally to me. I see that Box 1 contains $A$ for Pablo and $B$ for Penelope, while Box 2 contains $B$ for Pablo and $A$ for Penelope, plus an extra dollar for each. Then you invite me to choose one of the boxes so as to benefit the twins in the specified way.

Table 11.5 depicts our decision problem in tabular form, where $A+$ can be interpreted as the outcome $A$ plus one dollar, and $B+$ can be interpreted similarly. Here the columns represent the independent criteria 
Table 11.5 Two transparent boxes

\begin{tabular}{lcc}
\hline & column $1-$ Pablo $(0.5)$ & column $2-$ Penelope $(0.5)$ \\
\hline Box 1 & $A$ & $B$ \\
Box 2 & $B+$ & $A+$ \\
\hline
\end{tabular}

or sources of value - Pablo's and Penelope's respective welfare - that matter (equally, by assumption) to the decision-maker. As noted earlier, "Two Transparent Boxes" is constructed to have the same tabular form as Hare's "Two Opaque Boxes" problem, the difference being the meaning of the columns. In Hare's problem, the columns represent rather the possible states of the world (each equi-probable by assumption since they are the results of a fair coin toss). (A further difference then is that the problem in Table 11.5 involves two dollar coins, while Hare's problem requires just one for which the accompanying prize is uncertain.) In both problems it is assumed that $A+$ is strictly preferred to $A, B+$ is strictly preferred to $B$, and $A$ and $A+$ are each incommensurable with $B$ and $B+$, independent of the column, whether the criterion or the supposed state of the world.

The decision problem depicted in Table 11.5 - whether the transparent or opaque version - can be regarded as having the same crucial "complementarity" in expected utility values across the columns as the final geoengineering problem above. Assume that the incommensurability between $A$ and $B$ is represented by a set of expected utilities, some of which put $A$ higher in value and some of which put $B$ higher in value. Recall that when a dollar is added to either prize, the two remain incommensurable. On some expected utility functions then, $A$ is better than $B$ plus a dollar, favoring Box 1 in the first column. But on those very same expected utility functions, $A$ plus a dollar is better than $B$, by an even greater amount. So Box 1 loses by a greater amount in the second column than it wins in the first column. Likewise, for the expected utility functions in which Box 1 wins on the second column, it loses by a greater amount on the first column.

By the weak (criterion-wise) competitiveness principle, both Box 1 and Box 2 are permissible choices in the Two Transparent Boxes problem. Similarly, by weak (state-wise) competitiveness, both boxes are permissible choices in the Two Opaque Boxes problem. That is because, under each of the columns (i.e., each of the criteria/states), the two options are incommensurable. Hence each option is not worse than the other across all the columns and thus each option is competitive. But surely, whichever problem one faces, the choice of Box 2 is better! Moreover, our utility model described above - revealing the "complementarity" between the column utilities (to the detriment of Box 1) for all possible utility functions apparently reveals why it is that Box 2 comes out determinately better. 


\section{Katie Steele}

Some argue that Box 1 in "Two Opaque Boxes" is nonetheless a permissible choice (for instance, Bales et al. 2014). The major positive argument for this claim (noted already by Hare, 2010) involves appealing to the agent's attitudes were she fully informed. In "Two Opaque Boxes" the agent may reason that there are two ways in which the world might go, corresponding to the two states of the world. Or, put differently, if she were fully informed, there are two possible states of knowledge she might have. Either way, her fully informed self will not find Box 1 worse than Box 2 (in fact, she will find them to be incommensurable). So why not defer to her fully informed self and regard the two boxes as permissible choices, neither one worse than the other, even under uncertainty? The general principle might be stated as follows:

Defer to Fully Informed Self. If you know that your fully informed self will find an option no worse than alternatives, then you should now, even under uncertainty, find this option no worse than alternatives and thus permissible.

Something along the lines of the aforementioned is typically granted in the literature as a reason to endorse the weak (state-wise) competitiveness principle. The dispute is about whether this is a good enough reason to overcome the reasons against endorsing the weak (state-wise) competitiveness principle. But we need not here take a side in this debate (see, however, Rabinowicz, 2021, for further analysis). ${ }^{6}$ What is important for our discussion is that this reason in favor of weak (state-wise) competitiveness does not translate well to the multi-criteria setting. The analogue general principle is as follows:

Defer to Fully One-Track-Minded Self. If you know that your fully one-track-minded self will find an option no worse than alternatives, then you should now, even taking into account all criteria, find this option no worse than alternatives and thus permissible.

In the case of "Two Transparent Boxes," the idea is that when the decision-maker focuses on the respective plights of Pablo and Penelope (the "criteria" in this decision problem), she finds neither box worse than the other. Hence, she should find neither box worse than the other overall when all criteria are taken into account.

The crucial problem with this proposed maxim to defer to one's fully one-track-minded self is that this self (or rather these selves), unlike one's fully informed self (or possible selves), has no claim to authority. It simply does not matter what would be my attitudes were I to only care about any single aspect or criterion of choice. That has nothing to do with either my actual or idealized preference attitudes. 
These considerations bear on the question of whether "complementarities" in candidate expected utilities across different columns (criteria/ states) of a decision problem matter or are even recognized as meaningful. The representation of the agent's attitudes in "Two Transparent Boxes" described earlier, whereby on any candidate expected utility function, Box 2 is better for one person (criterion) by a greater amount than it is worse for the other person (criterion) denies any special primacy to criteria taken in isolation. The idea is that it is simply a further fact about the agent's attitudes that Box 2 is better than Box 1 overall, despite the two boxes being incommensurable with respect to each of the criteria taken in isolation.

Compare the situation with "Two Opaque Boxes." Some argue that here too, there may be facts about the agent's attitudes, to do with "complementarities" in their expected utilities across states, that cannot necessarily be discerned by looking at the states in isolation. Jim Joyce (2010) emphasizes this point in discussing agents who are intuitively best modeled as having "imprecise probabilities." Certain decision problems strongly suggest that the agent considers a number of possible probability functions as live candidates and that complementarities across states, or patterns that are true of all these candidate probability functions, are real and important. ${ }^{7}$ But at least in the case of states, it is plausible to argue for the contrary position: that one's attitudes with respect to states taken in isolation are basic, these being the possibilities for one's fully informed self, and that one's overall attitudes across states are derived from one's attitudes toward the states in isolation. In other words, it is plausible to deny any significance to the complementarities or global features shared by all the candidate probability and utility function pairs that may play a role in modeling one's preference attitudes across states. It follows that there is reason to think weak (state-wise) competitiveness is a constraint on rational preference. There are no such reasons favoring weak (criterion-wise) competitiveness.

\section{Return to Ordinary Decision-Making Practices}

A case has thus been made against choice principles based on a nondominance relation for the multi-criteria decision setting that extends to incommensurability. The principles in question were dubbed weak/strong (criterion-wise) competitiveness. Any stronger principle - such as a version of independence that extends to incommensurability - is thus also undermined by the analysis here. Recall that the rough idea of the stronger principle is "ignore those aspects of options whereby they are incommensurable, and base one's overall preferences on the remaining criteria." This is a poor guide to choice deliberations. We have seen that an option cannot be ruled out of contention just because it is dis-preferred on all those criteria for which it is not incommensurable with another option. 


\section{Katie Steele}

Moreover, an option that is preferred or at least not dis-preferred on all those criteria for which it is not incommensurable with another option may nonetheless be dis-preferred overall.

Are our ordinary choice deliberations then routinely unreasonable? For all that was said in Section 1, the answer is apparently "yes." The Bike Route decision problem was introduced to motivate the idea that we do indeed routinely ignore those aspects of options or criteria for which we cannot compare options. And this decision problem is as mundane as any.

There is, however, a more charitable reading of our ordinary choice deliberations in light of the findings here. It is to insist that appearances can be deceiving. To the extent that we do "ignore incommensurabilities" in assessing options, it is because the incommensurabilities in question are relatively "minor"; they do not "swamp" those aspects of the options for which the agent has clear preferences (as per the decision problem in Table 11.2). In other cases, we may not be ignoring incommensurabilities at all but rather doing something which, again, is typically regarded as faultless (and in accordance with the model of multi-criteria decisionmaking presupposed in this discussion): we may simply be ignoring criteria for which we are indifferent between the options.

The more charitable reading raises further questions, however, about the reasonableness of ordinary choice deliberations. The deliberations as described may well be consistent, in the sense of according with the appropriate preference axioms. But one might doubt whether it is reasonable in a more substantive sense to be (more or less) indifferent between options with respect to criteria that are less salient, say, criteria concerning the options' more complicated causally downstream effects. ${ }^{8}$ For instance, one might doubt whether it is reasonable to be indifferent between the city bike route and the green bike route when it comes to complicated downstream effects like one's impact on the people one will encounter along the route and how these people will in turn impact on those they encounter and so on.

Here the discussion prompted by James Lenman's worry that we are "clueless" about the consequences of our actions is helpful. Hilary Greaves (2016) offers a limited defense of our being indifferent between pairs of options with respect to their more complicated causally downstream effects. The defense rests on a specific application of "the principle of indifference": roughly that in many cases, there is no reason to think that some coincidental downstream effect of one option will not be equally likely to be a coincidental downstream effect of the other option instead.

The question is whether an appeal of this sort to the principle of indifference is typically apt. Greaves (2016) herself outlines circumstances in which it is not: cases where conflicting considerations bear on how options are ranked with respect to the more difficult choice criteria concerning the options' causally downstream effects. Perhaps Laurie Paul has something like this in mind with respect to decisions involving transformative 
experience: for many choice criteria associated with life satisfaction, say, there will be unresolved conflicting considerations regarding how the options should be ranked. In such cases, the reasonable attitude is arguably one of incommensurability between the options under the criteria in question. But note that there seems no reason for this incommensurability, in general, to be relatively minor, such that it can be safely ignored. Quite the opposite. The incommensurability here seems significant, and if the choice criteria in question are also significant, then the incommensurability will have a "swamping" effect. That is, to the extent that our decision problems are best described in the way just outlined, then we must live with widespread indeterminacy or incommensurability in our overall ranking of options.

\section{Notes}

1 The reason for this incompleteness of preference or lack of opinion would presumably be that the criterion in question is itself multi-dimensional. For example, the comfort of other riders may depend on both companionship and ease of riding; these may pull in opposite directions, and it may be unclear how they trade-off against each other.

2 Compare with Bykvist and Stefánsson's (2017) similar interpretation of Paul's proposal for making decisions involving transformative experience. (They go on to critique the proposal.)

3 Note that Rabinowicz (2021) rejects the state-wise competitiveness principles stated here (in line with Hare), but both regard the principles to be at least plausible, if ultimately incorrect.

4 Typically, sets of expected utility representations are thought to be convex, but the appeal to discrete, non-convex sets does not compromise the analysis here.

5 See, for instance, Keeney and Raiffa (1976) for a representation theorem supporting this choice rule, at least when the preference orderings concerned are complete. In our examples, some preference orderings for criteria are assumed incomplete. But Keeney and Raiffa's theorem may nonetheless be taken to provide support for the weighted average choice rule, even a modified version that yields sets of weighted averages. And we can suppose that there is independent support for the representation of the preferences under each criterion as given in the tables.

6 Several reasons are given against endorsing weak (state-wise) competitiveness. Hare (2010) notes that there is a consideration in favor of Box 2, the dollar, but none in favor of Box 1. Moreover, Hare appeals to an argument from transitivity that is made explicit by Rabinowicz (2021): if one switches the prizes for Box 2 between the columns in Table 11.5, this option dominates and is thus better than Box 1. Surely one should be indifferent between ordinary Box 2 (as it appears in Table 11.5) and the switched Box 2. But then, by transitivity, ordinary Box 2 is better than Box 1 . To deny this, and thus maintain that Box 1 is not worse than ordinary Box 2 and thus a permissible choice, one would have to resist the indifference of the ordinary and switched Box 2 or else admit a violation of transitivity.

7 The term "complementarity" used here is indeed taken from Joyce, although he uses it in a narrower sense and with respect to candidate probability functions. 


\section{Katie Steele}

8 The phrase "(more or less) indifferent" is intended to account for cases of "minor incommensurabilty" (as discussed earlier and illustrated in Table 11.2 ), as well as cases of genuine indifference.

\section{References}

Bales, A., Cohen, D. and Handfield, T. (2014), 'Decision Theory for Agents with Incomplete Preferences', Australasian Journal of Philosophy 92/3: 453-470.

Bykvist, K. and Stefánsson, H.O. (2017), 'Epistemic Transformation and Rational Choice', Economics and Philosophy 33: 125-138.

Greaves, H. (2016), 'Cluelessness', Proceedings of the Aristotelian Society, 116/3: 311-339.

Hare, C. (2010), 'Take the Sugar', Analysis 70/2: 237-247.

Joyce, J.M. (2010), 'A Defense of Imprecise Credences in Inference and Decision Making', Philosophical Perspectives 24: 281-323.

Keeney, R.L. and Raiffa, H. (1976), Decisions with Multiple Objectives: Preferences and Value Tradeoffs (Wiley).

Lenman, J. (2000), 'Consequentialism and Cluelessness', Philosophy \& Public Affairs 29/4: 342-370.

Paul, L.A. (2014), Transformative Experience (Oxford University Press).

Rabinowicz, W. (2021), 'Incommensurability Meets Risk', in H. Andersson and A. Herlitz (eds.), Value Incommensurability: Ethics, Risk and Decision-Making (Routledge). 